\title{
New Stability Criteria for the Design of Low-Pass Sigma-Delta Modulators
}

\author{
J.A.E.P. van Engelen and R.J. van de Plassche \\ Department of Electrical Engineering, Eindhoven University of Technology \\ P.O.Box 513, 5600 MB Eindhoven, the Netherlands \\ J.A.E.P.v.Engelen@ele.tue.nl
}

\begin{abstract}
This paper describes a new model for the stability analysis of low-pass Sigma-Delta modulators $(\Sigma \Delta \mathrm{Ms})$ using the describing function method. The transfer of a single-bit quantizer is represented by a global signal gain and a phase uncertainty. This phase uncertainty arises from the limited accuracy in time with which the quantizer can detect the quantization-level crossings. This new model allows for a better prediction of possible idle patterns (important for the in-band signal-to-quantization error ratio), and calculation of zero-input stability boundaries for loop-filter parameters in higher order low-pass $\Sigma \Delta$ Ms.
\end{abstract}

\section{INTRODUCTION}

Sigma-Delta $(\Sigma \Delta)$ modulation [1] has become a very useful and popular technique for high-precision A/D conversion of narrow-band signals. As with other systems based on negative error feedback, one of the basic methods of improving the resulting (in-band) signal-to-error ratio is to increase the order of the loop-filter. Higher order, high-gain filters in feedback-loops can however lead to instability of the system. Instability in sampled systems usually manifests itself in the occurrence of limit cycles. Some of these limit cycles (called idle patterns) are imperative for the correct operation of the system. In practice we distinguish idle patterns and large signal limit cycles:

Idle patterns: Limit cycles with a small resulting (stable) amplitude, that are easily disturbed by applying an input to the system. The frequencies of these cycles usually are located far away from the signal-band, in which the loopgain usually is very high, rendering small amplitude signals impossible.

Large signal limit cycles: Limit cycles with a large resulting (stable or even continuously growing) amplitude, that therefore are hard (or impossible) to disturb. The frequencies of these cycles are often located near the signal-band.

To analyze the stability of sigma-delta modulators $(\Sigma \Delta \mathrm{Ms})$ we will consider the general discrete-time model as depicted in Figure 1, consisting of loop-filter $G$ and quantizer $Q$.

This work was supported by Philips Research Labs, Eindhoven, the Netherlands under project number RWC-061-ps-940028-ps.

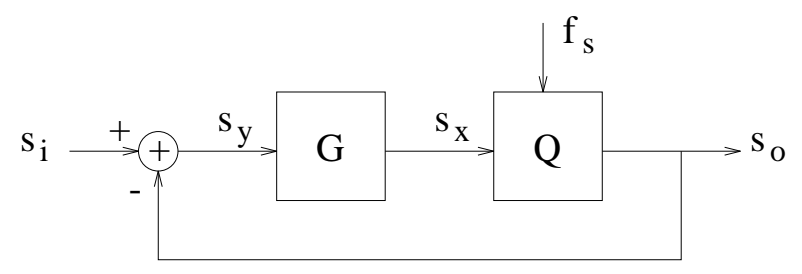

Fig. 1. Block diagram of a general Sigma-Delta modulator.

A well-known and commonly used method to analyze the stability of non-linear feedback systems is the describing function method [2]. Examples of the application of this method to $\Sigma \Delta \mathrm{Ms}$ can be found in [3] and [4]. The main problem using this method is the ability to adequately model the non-linear element $Q$. Generally the quantizer is modeled as a global signal gain (which we will call $\lambda$ ). For a single-bit quantizer this (real-valued) gain can range from $\lambda=0$ if the input signal is extremely large to $\lambda=\infty$ if the input signal is zero. A sampled quantizer (and a single-bit quantizer in particular) introduces a phase uncertainty [5] that can have a considerable influence on the overall stability of the system. Modeling of this phase uncertainty allows for a better prediction of possible idle patterns (important for the in-band signal-to-quantization error ratio), and calculation of zeroinput stability boundaries for loop-filter parameters. This will be shown in the following sections.

\section{Phase UnCERTAinty}

Phase uncertainty in the signal-transfer of sampled quantizers arises from the limited accuracy in time with which a quantizer can detect the quantization-level crossings of the input signal. The phase uncertainty of the single-bit quantizer does not depend on the amplitude of the input-signal as there is only one quantization level. This allows us to model the gain and the phase uncertainty in the quantizer independently.

The maximum absolute inaccuracy in time with which a single-bit quantizer can detect the zero-crossings of an input sine-wave with period $T$, is equal to half the minimum distance in time between these zero-crossings occuring at $l \frac{T}{2}$ and the sampling times occuring at $k T_{s}=k / f_{s}$ not coinciding with the zero-crossings:

$$
\frac{1}{2} \min _{k, l}\left|k T_{s}-l \frac{T}{2}\right|, \quad \text { with: } k T_{s} \neq l \frac{T}{2}
$$

which can be simplified to: $\frac{1}{2} \min \left(T_{S}, \frac{T}{2}-T_{S}\right)$. As shown in 


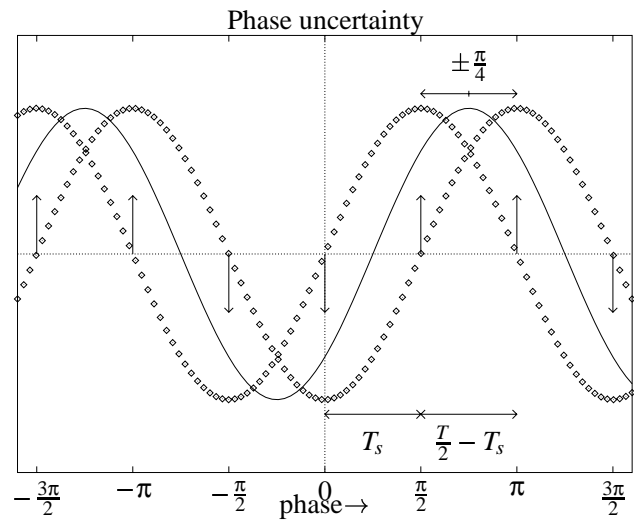

Fig. 2. Phase uncertainty of a single-bit quantized and sampled sine-wave with frequency $\frac{f_{s}}{4}$

Figure 2, an input signal (solid line) can be shifted in time, without changing the output of the quantizer (impulses). For an input frequency of $\frac{f_{s}}{4}$, as shown here, the maximum shift in time equals $\pm \frac{T_{s}}{2}$, corresponding to a phaseshift of $\pm \frac{\pi}{4}$. As a result of this maximum shift in time, the maximum phase uncertainty between input and output of the quantizer can be written as function of the normalized frequency $\theta=2 \pi f T_{s}=$ $2 \pi T_{S} / T$ of the input signal (Fig. 3):

$$
\Delta \phi(\theta)_{\max }= \pm \frac{1}{2} \min (\theta, \pi-\theta) .
$$

In order to model this phase uncertainty of which the range depends on the input signal frequency (Fig. 3), we introduce a new function using a dimensionless variable $\alpha$ :

$$
\Delta \phi_{\alpha}(\theta)=\alpha\left(\left|\theta+\frac{\pi}{2}\right|-\left|\theta-\frac{\pi}{2}\right|-\theta\right) .
$$

When using $\alpha$, the linearized global transfer $\tilde{Q}(z)$ can be described by the global gain $\lambda$ and this phase uncertainty $\Delta \phi_{\alpha}(\theta)$ with $0<\lambda<\infty$ and $-\frac{1}{2}<\alpha<\frac{1}{2}$ :

$$
\tilde{Q}(z)=\lambda \cdot e^{j \Delta \phi_{\alpha}(\theta)}, \quad \text { with: } z=r \cdot e^{j \theta} .
$$

Choosing $\alpha=0$ reduces (3) to the commonly used model of a (real-valued) global gain $\lambda$.

As the phase uncertainty becomes very small for small $\theta$ and the global transfer then approximates $\lambda$, one might expect this extension to have very little influence on the stability criteria of low-pass $\Sigma \Delta$ Ms. However, in the following section we will show that this extended model allows for a better prediction of idle patterns and calculation of zeroinput stability boundaries for loop-filter parameters.

\section{StabiLity}

When using the model for the quantizer derived in the previous section, the linearized signal transfer of the system (Fig. 1) is written as

$$
\widetilde{\left(\frac{S_{o}}{S_{i}}\right)}(z)=\frac{\lambda e^{j \Delta \phi_{\alpha}(\theta)} G(z)}{1+\lambda e^{j \Delta \phi_{\alpha}(\theta)} G(z)}, \quad \text { with: } z=r e^{j \theta} .
$$

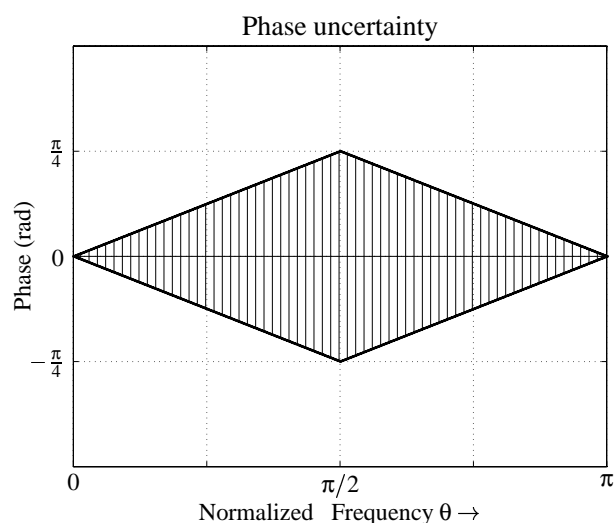

Fig. 3. Phase uncertainty of a single-bit quantizer.

The poles of the system, determining the stability of the system, are equal to the solutions of the stability equation

$$
1+\lambda e^{j \Delta \phi_{\alpha}(\theta)} G(z)=0, \quad \text { with: } z=r e^{j \theta} .
$$

The solutions of this equation are categorized by their positions in the complex plane:

- All poles of the system are inside the unit circle in the complex $z$-plane: The system is stable.

- One or more poles are on the unit circle, and the poles will be moved towards the origin by applying or increasing an input: The system contains idle patterns.

- One or more poles are on the unit circle, and poles will be moved away from the origin by increasing the input: The system is on the verge of instability.

- One or more poles are outside the unit circle: The system is unstable.

\section{A. Idle patterns}

Prediction of idle patterns is important, because if small inputs disturb the idle pattern this will result in the coding of the signal with an idle frequency. In the case that the actual idle frequency is lower, this effectively results in a lower coding frequency, a lower oversampling ratio, and thus in higher in-band quantization errors.

In the case of idle patterns, one or more poles are on the unit circle $\left(z=e^{j \theta}\right)$ and can be calculated by reducing (5) to:

$$
1+\lambda e^{j \Delta \phi_{\alpha}(\theta)} G\left(e^{j \theta}\right)=0 .
$$

In order to determine which idle frequencies are possible, this equation can be reduced to a phase criterion, as $\lambda$ is positively real-valued:

$$
\Delta \phi_{\alpha}(\theta)+\operatorname{Arg}\left\{G\left(e^{j \theta}\right)\right\}-\pi=0 .
$$

Graphically, this corresponds to drawing the phase shift of the loop-filter $\operatorname{Arg}\left\{G\left(e^{j \theta}\right)\right\}$ minus $\pi$, applying the phase uncertainty from Figure 3 as an error-band, and verifying whether the zero phase shift falls within the error-band. For example, for a second order low-pass $\Sigma \Delta \mathrm{M}$ with loop-filter

$$
G(z)=\left(2 z^{-1}-z^{-2}\right) /\left(1-2 z^{-1}+z^{-2}\right),
$$




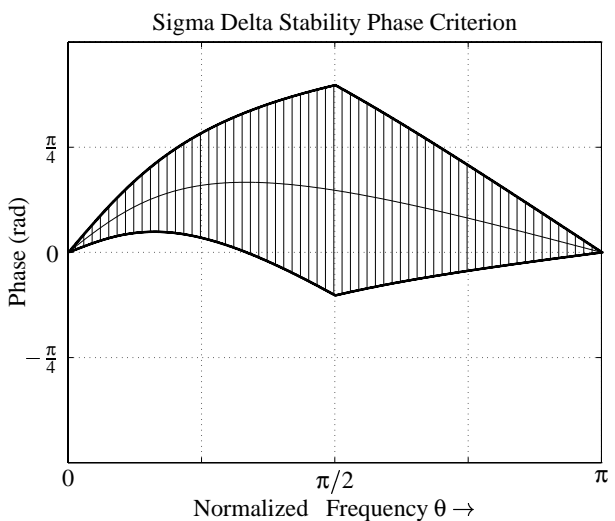

Fig. 4. Phasecriterion of a second order low-pass $\Sigma \Delta$ (8).

idle frequencies from $\theta \approx \frac{\pi}{3}$ to $\theta=\pi$ are possible (Fig. 4).

As the output of the single-bit quantizer can only have two values, idle frequencies equal to a sub-multiple of the sample frequency $f_{s}$ are possible. In the case of a low-pass $\Sigma \Delta \mathrm{M}$ odd sub-multiples are not allowed, because the idle patterns cannot contain a DC-value. For the second-order low-pass $\Sigma \Delta \mathrm{M}$ described here, idle frequencies of $\frac{f_{s}}{2}$ and $\frac{f_{s}}{4}$ are possible. The latter frequency is not predicted without modeling the phase uncertainty. Both idle patterns were observed in both simulations and experiments using the test set-up described in section IV.

\section{B. Zero-input stability}

A basic method to overview the behaviour of the system is to draw the poles of the system (i.e. the roots of (5)) in the complex plane, as a function the global gain $\lambda$. Modeling the phase uncertainty with a second parameter converts pole trajectories into a continuous set of trajectories (an area), making the root locus plot hard to evaluate. Therefore the trajectories are plotted as a function of $\lambda$ for discrete values of $\alpha$. The basic thought behind this is that in low-pass $\Sigma \Delta \mathrm{Ms}$ the input amplitude of the quantizer is minimized, effectively changing $\lambda$, but leaving $\alpha$ unchanged. We will now apply this procedure to two cases.

Case I: First we analyze a second order $\Sigma \Delta \mathrm{M}$ described by (8). The root locus is shown in Figure 5 for several values of $\alpha$.

A discrete-time system is stable when all poles of the system are inside (or on, in the case of a stable oscillation) the unit circle in the complex plane. For zero-input stability, this means a non-empty set of initial conditions exists for which the poles of the system should either stay inside the unit circle, or move into the unit circle within a finite period of time. In practice, the range of initial conditions should be large enough to allow for a meaningful operation of the system. The range of initial conditions results in a range of initial values for $\lambda$ and $\alpha$. Generally, the initial condition values (and thus the input of the quantizer) are very small, resulting in a large value for $\lambda$. In this case one or two poles will be outside the unit circle (Fig. 5). Due to the single-bit quantizer, which always has a non-zero output value, a signal with increasing amplitude will appear in the system. This will reduce $\lambda$ and move the poles into or onto the unit circle for every possible value of $\alpha$ : the system becomes stable for all initial conditions. Depending on the actual value of $\alpha$, one of two stable operating points results, as predicted in the previous section:

- $\alpha=0$ : A single pole on the unit circle for $z=-1$, corresponding to an idle pattern at $f_{s} / 2$.

- $\alpha \approx-0.295$ : A pair of conjugate poles on the unit circle for $z= \pm j$, corresponding to an idle pattern at $f s / 4$.

Case II: We now apply this method to a class of higher order ( $>3$ ) low-pass $\Sigma \Delta$ Ms described by the class of loopfilters ${ }^{1}[6]$ :

$$
G(z)=\frac{(z-a)^{n}}{(z-1)^{n}}-1, \quad \text { with: }\left\{\begin{array}{l}
n=3,4,5, \ldots \\
-1<a<1
\end{array} .\right.
$$

When modeling the quantizer as an addition of (inputindependent) quantization errors, the transfer of this error signal to the output of the $\Sigma \Delta \mathrm{M}$ (Fig. 1) equals

$$
\frac{1}{1+G(z)}=\frac{(z-1)^{n}}{(z-a)^{n}}
$$

revealing the low-pass $\left(z=e^{j 0}=1\right)$ error-shaping characteristic of these $\Sigma \Delta$ Ms. In Figure 6 the root locus is shown for a third order $(n=3) \Sigma \Delta \mathrm{M}$ with $a=0$. For large values of $\lambda$, one or two poles will be outside the unit circle. The resulting increasing amplitude of the signal in the system effectively decreases $\lambda$. For some values of $\alpha$ the poles will not enter the unit circle until $\lambda=0$ : the $\Sigma \Delta \mathrm{M}$ is not zero-input stable for a considerable range of initial conditions.

\footnotetext{
${ }^{1}$ The second order loop-filter described by (8) also belongs to this class: $a=0$ and $n=2$.
}

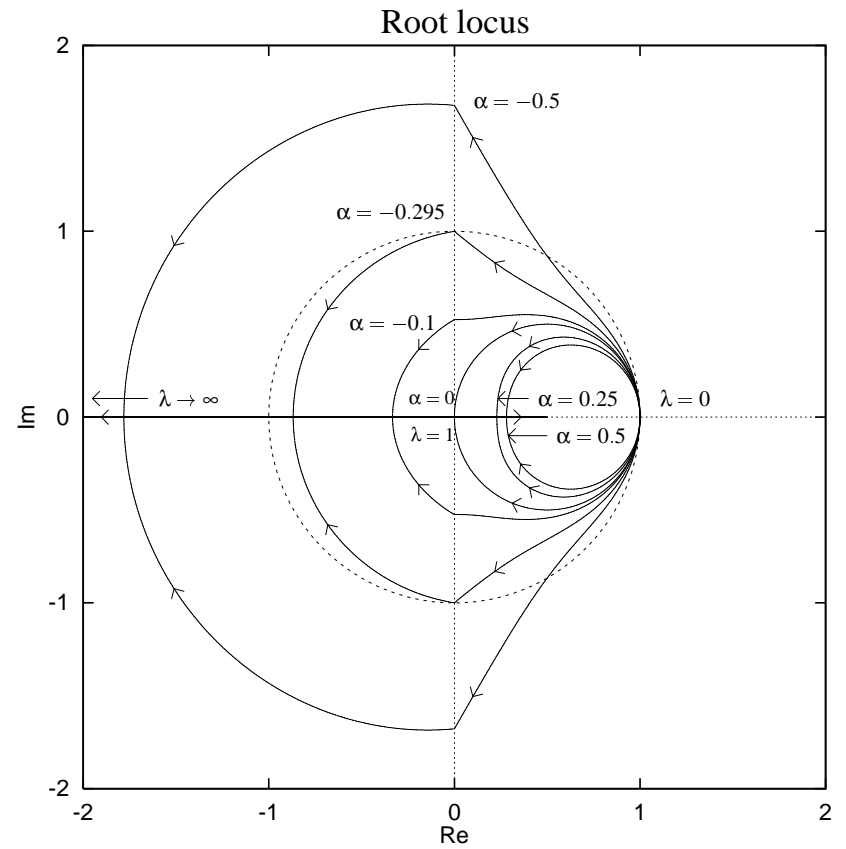

Fig. 5. Root locus of a second order low-pass $\Sigma \Delta \mathrm{M}$ described by (8) for different values of the phase uncertainty parameter $\alpha$. 


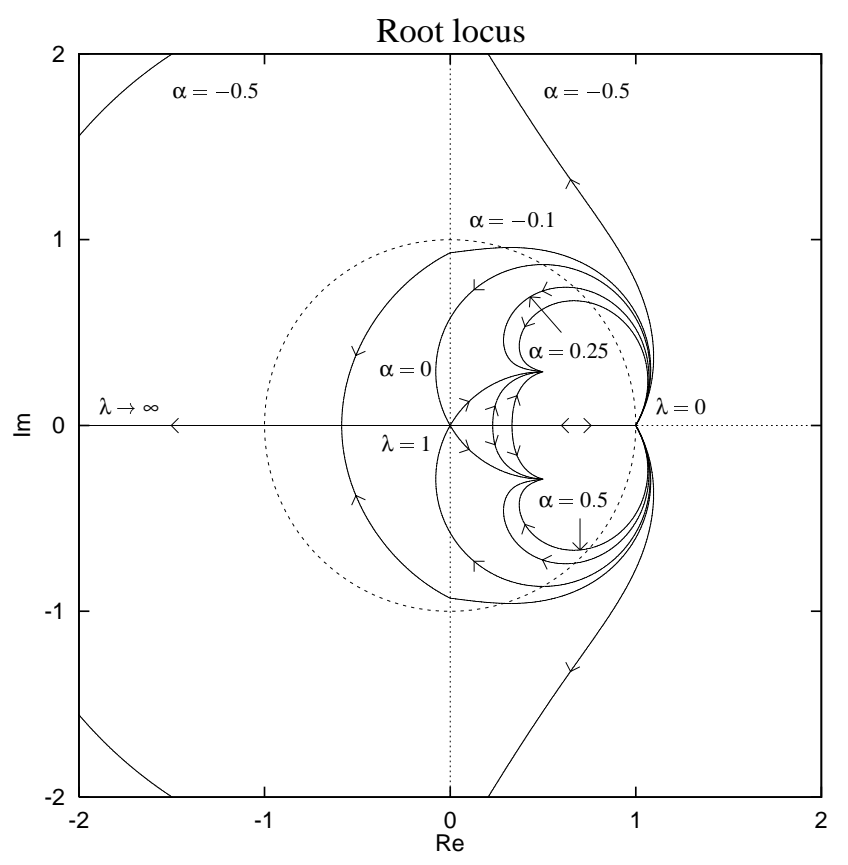

Fig. 6. Root locus of a third order low-pass $\Sigma \Delta M(a=0)$ for different values of the phase uncertainty parameter $\alpha$.

In order for the $\Sigma \Delta \mathrm{M}$ to be zero-input stable, the poles should enter the unit circle for every $\alpha$, with $\lambda>0$. As the root locus of the $\Sigma \Delta \mathrm{M}$ changes together with filter parameter $a$, a boundary (minimum) value for $a$ can be found for which the $\Sigma \Delta \mathrm{M}$ is zero-input stable. In Figure 7 the root locus with $\alpha=-\frac{1}{2}$ (corresponding to the outermost trajectory possible) is plotted for different values of the filter parameter $a$. For $a \gtrsim 0.41$ the trajectories intersect the unit circle for all $\alpha$ at $\lambda>0$ and the system is zero-input stable. The root loci for $n=4,5, \ldots$ are similar the one shown in Figure 6 , and minimum values for $a$ can be obtained in the same way as for the third order $\Sigma \Delta \mathrm{M}$. The minimum values for $a$ can be calculated numerically by determining the value for which the root locus for $\alpha=-\frac{1}{2}$ is tangent to the unit circle. These calculated minimum values for $a$ are listed in Table I, together with minimum values determined experimentally needed for zero-input stability. The differences between experimental and calculated values are due to the linearization in modeling the single-bit quantizer.

TABLE I

MinimAL VALUES FOR $a$ FOR WHICH THE SYSTEM DESCRIBED BY (9) IS ZERO-INPUT STABLE FOR ORDER $n$.

\begin{tabular}{|c|c|c|}
\hline$n$ & \multicolumn{2}{|c|}{$a$} \\
\hline & numerical & experimental \\
\hline 3 & 0.412 & 0.415 \\
4 & 0.587 & 0.616 \\
5 & 0.679 & 0.713 \\
6 & 0.736 & 0.769 \\
\hline
\end{tabular}

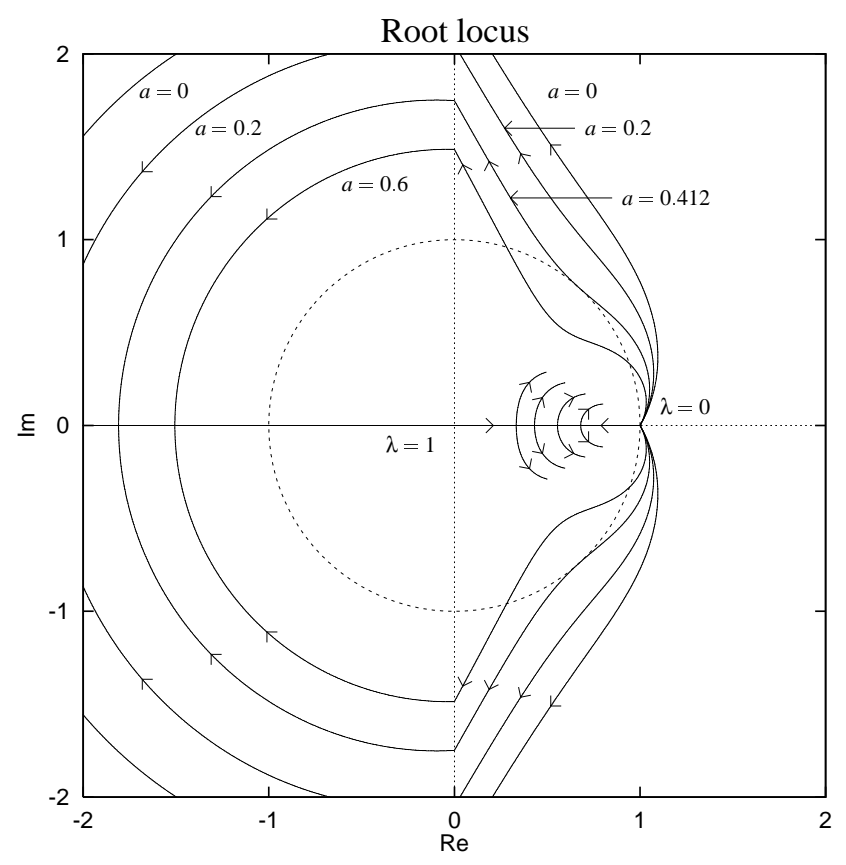

Fig. 7. Root locus $\left(\alpha=-\frac{1}{2}\right)$ of a third order low-pass $\Sigma \Delta M$ for different values of the filter parameter $a$.

\section{TEST SET-UP}

In order to verify the theoretical results both computersimulations and an experimental test set-up were used. The test set-up consisted of a 12-bit A/D converter (Philips TDA 8768) frontend, a 10-bit D/A converter (Philips TDA 8776) backend and a programmable logic device (Altera EPF81500) containing 1500 flipflops and over 15000 ports. The programmable logic device was used to implement all-digital $\Sigma \Delta \mathrm{Ms}$ with 28-bit wide internal registers and upto four adders per filter coefficient. The filter coefficients were implemented using Canonical Signed Digit (CSD) encoding. This set-up allows for real-time evaluation of the behaviour of digital $\Sigma \Delta \mathrm{Ms}$ for speeds up to $10 \mathrm{MHz}$. Digital implementation of the $\Sigma \Delta \mathrm{M}$ makes exact control of system parameters possible; the use of an analog frontend and backend simplifies control and evaluation of input and output signals.

\section{Conclusions}

A new model for the stability analysis of low-pass SigmaDelta modulators $(\Sigma \Delta \mathrm{Ms})$ is described. The analysis is based on the describing function method in which the non-linear element is modeled by a global transfer. The single-bit quantizer of the $\Sigma \Delta \mathrm{M}$ is modeled by a global gain and a variable phase uncertainty. This new model can be reduced to the commonly used model of a global gain by settting the phase uncertainty model parameter to zero. This extended model allows for a better prediction of possible idle patterns (important for the in-band signal-to-quantization error ratio), and calculation of zero-input stability boundaries for loop-filter parameters in low-pass $\Sigma \Delta \mathrm{Ms}$. Both results are not obtained without modeling the phase-uncertainty of the quantizer. 


\section{REFERENCES}

[1] J.C. Candy and G.C. Temes, "Oversampling methods for a/d and d/a conversion," in Oversampling Delta-Sigma Data Converters, J.C. Candy and G.C. Temes, Eds. IEEE Press, New York, 1991.

[2] D.P. Atherton, Stability of Non-Linear Systems, Research Studies Press; Wiley, 1981.

[3] R.T. Baird and T.S. Fiez, "Stability analysis of high-order delta-sigma modulation for adc's," IEEE Trans. on Circuits and Systems-II, vol. 41 no. 1, pp. 59-61, January 1994.

[4] S.H. Ardalan and J.J. Paulos, "An analysis of nonlinear behavior in delta-sigma modulators," IEEE Trans. on Circuits and Systems, vol. 34, no. 6, pp. 593-603, June 1987.

[5] M.H.H. Höfelt, "On the stability of a 1-bit-quantized feedback system," in Proc. ICASSP, Washington, 1979, pp. 844-848.

[6] Eduard F. Stikvoort, "Some remarks on the stability and performance of the noise shaper or sigma-delta modulator," IEEE Trans. on Communications, vol. 36, no. 10, pp. 1157-1162, October 1988. 